\title{
A novel integrative method for analyzing eye and hand behaviour during reaching and grasping in an MRI environment
}

\author{
Jane M. Lawrence $\cdot$ Kamyar Abhari $\cdot$ Steven L. Prime • \\ Benjamin P. Meek • Loni Desanghere - Lee A. Baugh • \\ Jonathan J. Marotta
}

Published online: 18 March 2011

(C) Psychonomic Society, Inc. 2011

\begin{abstract}
The development of noninvasive neuroimaging techniques, such as fMRI, has rapidly advanced our understanding of the neural systems underlying the integration of visual and motor information. However, the fMRI experimental design is restricted by several environmental elements, such as the presence of the magnetic field and the restricted view of the participant, making it difficult to monitor and measure behaviour. The present article describes a novel, specialized software package developed in our laboratory called Biometric Integration Recording and Analysis (BIRA). BIRA integrates video with kinematic data derived from the hand and eye, acquired using MRI-compatible equipment. The present article demonstrates the acquisition and analysis of eye and hand data using BIRA in a mock (0 Tesla) scanner. A method for collecting and integrating gaze and kinematic data in fMRI studies on visuomotor behaviour has several advantages: Specifically, it will allow for more sophisticated, behaviourally driven analyses and eliminate potential confounds of gaze or kinematic data.
\end{abstract}

Keywords Software analysis package $\cdot$ Visuomotor processes - Eye-hand integration · Functional magnetic resonance imaging · Hand kinematics · Eye kinematics . Motion monitoring

J. M. Lawrence $(\bowtie) \cdot$ K. Abhari $\cdot$ S. L. Prime $\cdot$ B. P. Meek

L. Desanghere $\cdot$ L. A. Baugh $\cdot$ J. J. Marotta

Department of Psychology, University of Manitoba,

190 Dysart Road,

Winnipeg, Manitoba R3T 2N2, Canada

e-mail: lawrencj@cc.umanitoba.ca

\section{Introduction}

When we reach out to pick up an object, such as a glass of water or a pen, a complex series of actions are performed in order to complete this "simple" task. Our visual system must process information about the object, such as its shape, size, orientation, and location. These qualities are used to extend our arm and to preshape our hand to make an effective grasp (for a review, see Glover, 2004). Our understanding of how vision guides motor actions has been significantly advanced by behavioural studies that use eyeand hand-tracking methods in tandem (Carey, Della Sala, \& Ietswaart, 2002). Additionally, our understanding of visuomotor control has been further augmented by fMRI studies that have investigated the neural correlates of visuomotor behaviour (Culham, Cavina-Pratesi, \& Singhal, 2006). Integrating these two experimental paradigms would offer more comprehensive insight into the relationship between visuomotor behaviour and neural activity than either can when performed alone. As more MRI-compatible eye- and hand-tracking methods become available, neuroimaging research on visuomotor processes would greatly benefit from a reliable, affordable, and user-friendly system that records and integrates eye and hand movements.

Relative to nonimaging behavioural studies, fMRI investigations of visuomotor processes face two major limitations: the confined space within the bore of the MRI scanner and the presence of a strong magnetic field. Both of these factors limit the apparatus that can be used for presenting stimuli and collecting data on eye position and hand kinematics. One approach to deal with these limitations is to present participants with two-dimensional stimuli on a back-projected screen behind the scanner, which participants view through a mirror box attached to 
the head coil and interact with using an MRI-compatible computer mouse (Ogawa, Inui, \& Sugio, 2006), a joystick (Grefkes, Ritzl, Zilles, \& Fink, 2004), or a pneumatic robotic arm (Diedrichsen, Hashambhoy, Rane, \& Shadmehr, 2005). Other researchers have used MRI-compatible touch screens for stimulus presentation and data collection (Levy, Schluppeck, Heeger, \& Glimcher, 2007). However, in these studies, participants do not actually grasp an object, and therefore kinematic measures of the hand during a typical reaching or grasping movement (e.g., reach velocity, grip aperture, or reach trajectory) cannot be obtained.

To overcome this difficulty, fMRI investigations of visuomotor behaviour have utilized devices such as a pneumatically driven rotating barrel (Culham et al., 2003) or a sliding table (Marotta, Mraz, Black, \& Graham, 2007) to present graspable objects within an MRI environment. These devices have allowed researchers to move visuomotor paradigms, in which participants look, reach, or grasp threedimensional objects, into the magnetic field of the scanner. As with visuomotor investigations outside such a field, the participant is given a cue as to when and how to perform a given action. Generally, the time of the cue's presentation is used to identify a model of the expected task-related signal change in the general linear model analysis of fMRI data. An advancement of these paradigms that would allow for the simultaneous acquisition of combined kinematic and eye data would enhance fMRI studies of reaching and grasping by (1) allowing monitoring of task performance, (2) enabling quantitative analysis of behaviour, and (3) facilitating comprehensive data-driven fMRI analyses to contrast activations. If kinematic data were collected, behavioural recordings could be entered into a general linear model as covariates (either of interest in their own right or as confounds). To date, fMRI studies of reaching and grasping have not collected simultaneous kinematic and gaze data, and thus, activation differences between conditions could be due to confounds of kinematic measures (e.g., movement time) or gaze (foveal vs. peripheral).

The ability to measure eye and hand behaviour in the MRI environment has, in the past, been impeded by the same limitations that affect the study design. The simplest, and most common, method to observe motor behaviour in an MRI setting is to use a compatible video camera (e.g., Marotta et al., 2007; Rocca et al., 2007). However, the recent introduction of MRI-compatible motion-tracking systems that utilize infrared reflective markers (e.g., OQUIS MRI; Qualisys, Gotenberg, Sweden), fibre optic ribbon (e.g., ShapeClaw; Measureand Inc., Fredricton, Canada), and micro-electro-mechanical gyroscopes (Schaechter, Stokes, Connell, Perdue, \& Bonmassar, 2006) have improved the reliability and quality of movement data collected in an MRI environment. As new MRIcompatible devices capable of acquiring hand and eye movement data become more accessible, an easy method of integrating such equipment would represent a significant advancement in the field. The present article describes specialized software, the Biometric Integration Recording and Analysis (BIRA) program, that was designed to record, integrate, and analyse hand and eye kinematic data acquired simultaneously from two commercially available tracking systems, in addition to video-based recording. The ultimate goal of BIRA is not only to measure kinematic behaviour within an MRI field but also to use this information to strengthen and refine the statistical analysis of neuroimaging data. Before this can happen, we must first assess the overall behavioural performance of this new system. Here, we demonstrate BIRA's capability to simultaneously record and integrate reliable, quantitative measures of eyehand data in a simple reach-to-grasp task in a mock MRI.

\section{Method}

\section{Statement of ethics}

This study was approved by the Research Ethics Board of the National Research Council Institute for Biodiagnostics and the Psychology/Sociology Research Ethics Board of the University of Manitoba. Informed consent was obtained from participants prior to the study.

\section{General experimental paradigm}

Data were collected from 4 female, right-handed participants with normal or corrected-to-normal vision (average age: 29.75 years). Participants were positioned supine on the bed of a mock MRI scanner (0 Tesla) with their head in a mock head coil. Head motion was minimized by padding the head coil with extra foam wedges. An MRI-compatible sliding table was positioned on the bed at the participant's feet and extended over the participant's thighs so that objects were within reach of the right hand (Fig. 1). The participant viewed the table through a double-mirror box attached to the head coil. The double-mirror box ensured that the participant viewed the blocks in the correct, upright direction.

Three wooden rectangular blocks, $152 \mathrm{~mm}$ (wide) $\times 42 \mathrm{~mm}$ (high), $107 \mathrm{~mm} \times 60 \mathrm{~mm}$, and $80 \mathrm{~mm} \times 80 \mathrm{~mm}$, were individually presented to the participant by attaching them to a vertical canvas of Plexiglas $90 \mathrm{~mm}$ from the edge of the sliding table (Fig. 1). The blocks were securely attached to the Plexiglas canvas by fitting two pegs that extended from the canvas into holes in the backs of the blocks. A ruler was drawn along the width of the blocks' top and bottom back edges; the ruler was visible to an MRI-compatible video camera (MRC Systems, Heidelberg, Germany), but not to the participant. 

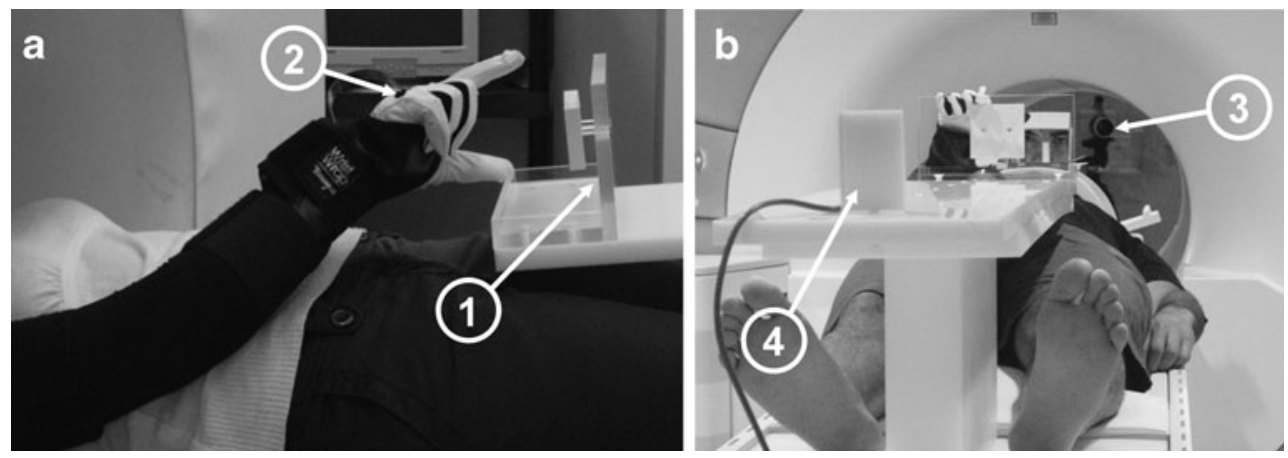

Fig. 1 Experimental setup of a participant reaching and grasping with ShapeClaw motion-monitoring tape mounted on the right arm. In a, (1) wooden blocks were held in place by two plastic pegs on the Plexiglas canvas secured to a sliding table. (2) Hand data were acquired via ShapeClaw fibre-optic tape attached to a glove worn on the participant's right hand. In $\mathbf{b}$, the objects were viewed through a double mirror box placed on the mock head coil. An infrared source was secured to the top of the mirror box to illuminate the right eye. (3) An SMI LR eye-tracking camera was located at the rear of the mock scanner, which captured eye movements through a single mirror. (4) Grasp positions on the block were verified by a video camera positioned behind the canvas and block
The visuomotor task began with the participant's eyes closed and the right hand held at a start position at the edge of the table with index finger and thumb together in a pinch formation. Participants were instructed, upon a verbal command, to open their eyes, grasp the block by its top and bottom edges using a pincer grasp with their index finger and thumb, return the hand to the start position, and close their eyes. This would be repeated 10 times.

Biometric Integration Recording and Analysis (BIRA) software package

BIRA is a specialized software package for recording, integrating, and analysing behavioural data acquired simultaneously from separate eye- and hand-tracking systems. BIRA was written in MATLAB 7.5.0 (R2007b) code and utilized MATLAB'S own built-in instrument control, image acquisition, and image-processing toolboxes (The MathWorks Inc., Natick, USA), as well as the separately available Psychophysics Toolbox (Brainard, 1997; Pelli, 1997). A copy of the BIRA source code is available for free by contacting us through our laboratory's Web site (www.perceptionandaction.com). BIRA was run on a Dell PC (Intel Core 2 Duo Processor 3.1 GHz, 4 GB RAM, Windows XP Professional SP3) and received behavioural data from the eye- and hand-tracking systems via a customized computer network system of three microprocessor-controlled personal computers (Fig. 2). BIRA is programmed to initiate data recording upon receiving a trigger from the MRI scanner. In the present study, this trigger was given by a manual keyboard press. Upon the trigger, BIRA begins recording and aligning incoming data as described below for each type of motion-tracking system.
Recording equipment used with BIRA in the present study

It is important to note that the BIRA software is easily adaptable to any motion-tracking systems (both present and future). In the present experiments, the motion-tracking systems used were dictated by availability and in no way reflect permanent parts of BIRA.

Hand kinematic data acquisition Hand kinematic data were obtained using ShapeClaw fibre optic ribbon and accompanying ShapeRecorder software (Measurand Inc., Fredricton, Canada) on a Dell PC (Intel Core 2 Duo Processor 3.1 GHz, 4 GB RAM, Windows XP Professional SP3). Hand position was recorded at a sampling rate of $100 \mathrm{~Hz}$. The ShapeClaw ribbon was positioned along the superior side of the right hand's thumb and index finger and held in place using a customized glove (labelled "2" in Fig. 1A). The remaining length of the

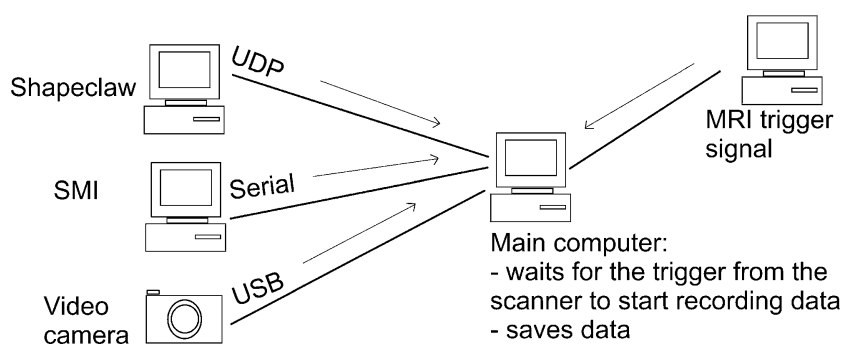

Fig. 2 Schematic of the customized computer network connecting the separate apparatus for tracking hand and eye movements, video data, and BIRA. The start of data acquisition in the BIRA software is triggered in this study by a keyboard press used to simulate an incoming trigger from an MRI scanner. Upon receiving the trigger, BIRA receives streaming hand data from ShapeClaw via a UDP connection, eye data from the SMI eye tracker via a serial connection, and video data from the camera via a USB connection 
ribbon ran up the participant's arm and was secured using an arm-length nylon sleeve with additional arm bands below the elbow and shoulder. Prior to data collection, hand data were calibrated as per the ShapeClaw system instructions (see the Appendix). Upon the initiation of data recording, hand data were streamed to the Dell PC on which BIRA was operating through a network connection.

Eye kinematic data acquisition Eye position data were acquired from the right eye using a monocular MRIcompatible video-based eye-tracking system (SensoMotoric Instruments, Teltow, Germany). The camera was positioned behind the scanner, aimed down the bore, and focused on a mirror box attached to the head coil, which provided a reflection of the participant's eyes (Fig. 1B). Eye data were recorded with a spatial resolution of $\sim 1^{\circ}$ and at a sampling rate of $60 \mathrm{~Hz}$. Prior to data collection, eye data were calibrated using a 13-point calibration board $(152 \mathrm{~mm}$ wide $\times 123 \mathrm{~mm}$ high, $1,280 \times 1,024$ pixel resolution). Upon the initiation of recording, data from the eye-tracking system were streamed to the Dell PC on which BIRA was operating via a serial connection.

Video acquisition An MRI-compatible video camera (MRC Systems, Heidelberg, Germany) was used to record the entire study. The video camera had an $8.8-\mathrm{mm}$ focal length, a resolution of $176 \times 144$ pixels, and a sample rate of $60 \mathrm{~Hz}$. The camera was secured on the top of the sliding table $42 \mathrm{~cm}$ behind the vertical Plexiglas canvas to obtain a clear view of the block and the participant's fingers at the final grasp position (Fig. 1B). The video signal was obtained via a USB connection through a cinch cable to a Hauppauge WinTV-HVR-950 video capture card connection to the master computer on which BIRA was operating.

Data analysis: General capabilities of BIRA

Before describing the analysis of sample data, we will first describe the general analysis methods and capabilities of BIRA. In addition to recording eye-hand data from multiple tracking systems, BIRA offers an integrative approach to analysing these data. Figure 3 shows a sample screen shot of the BIRA graphical user interface (GUI) during the analysis of eye-hand data from one typical trial in our reach-to-grasp task. The hand kinematic data (depicted by the line curve) and the timing of eye fixations (depicted by the shaded areas) are shown in the graphical display in the top half of the BIRA GUI (Fig. 3A). The video recording of that trial from the MRI-compatible video camera behind the block is shown in the display at the lower left portion of the GUI
(Fig. 3B). Spatial positions of the eye fixations are superimposed on a schematic of the stimulus (i.e., a rectangular wooden block) shown in the display in the lower middle portion of the GUI (Fig. 3C). BIRA can plot positional information of both eye and hand data onto any schematic stimulus specified by the experimenter. BIRA has the capability to plot eye and hand data together across different time epochs and allows the user to select data at any time point collected during the trial for detailed analysis. Detailed analyses of hand kinematic data in BIRA include reach velocity, position of thumb and index finger, grasp aperture, and timings of reach onset and end time. Grasp apertures were defined as the distance between the thumb and index finger. The analysis of eye position data in BIRA includes gaze position, number and location of fixations, and velocity of eye movements.

Hand kinematic data We analysed the final grasp position as determined by the horizontal locations of the index finger and thumb on the block, the maximum grip aperture during the reach movement (as determined by the distance between the index finger and thumb), and the maximum velocity during the reach. A moving average filter (55 samples) was applied to hand velocity profiles. Movement onset was determined by velocity profiles and confirmed with video.

Eye kinematic data Eye fixations were identified in BIRA using a dispersion algorithm (Salvucci \& Goldberg, 2000) with a duration threshold (100 ms) and a dispersion limit $(10 \mathrm{~mm})$. With these criteria, 1 participant did not exhibit fixations during grasping on Block D. Therefore, the eye kinematic data of this participant were excluded for all blocks, and all further analysis was conducted with the data from the remaining 3 participants. The locations of the first fixations for each of the trials were plotted in BIRA and their coordinates exported as a text file.

Video data To measure the position of the grasp location according to video data, a screen shot of each final grasp was acquired and saved as a bitmap image. Images were then examined using ImageJ (Abramoff, Magelhaes, \& Ram, 2004). The scale was set for each image by identifying the number of pixels between the centre and the 1-cm mark on the blocks. A grasp line was then drawn on the image from the centre of the index finger placement to the centre of the thumb placement. The distance from the horizontal center of the block to the grasp line was then measured at (1) the centre of the index finger, (2) the centre of the thumb, and (3) the level of the vertical centre of the block. 

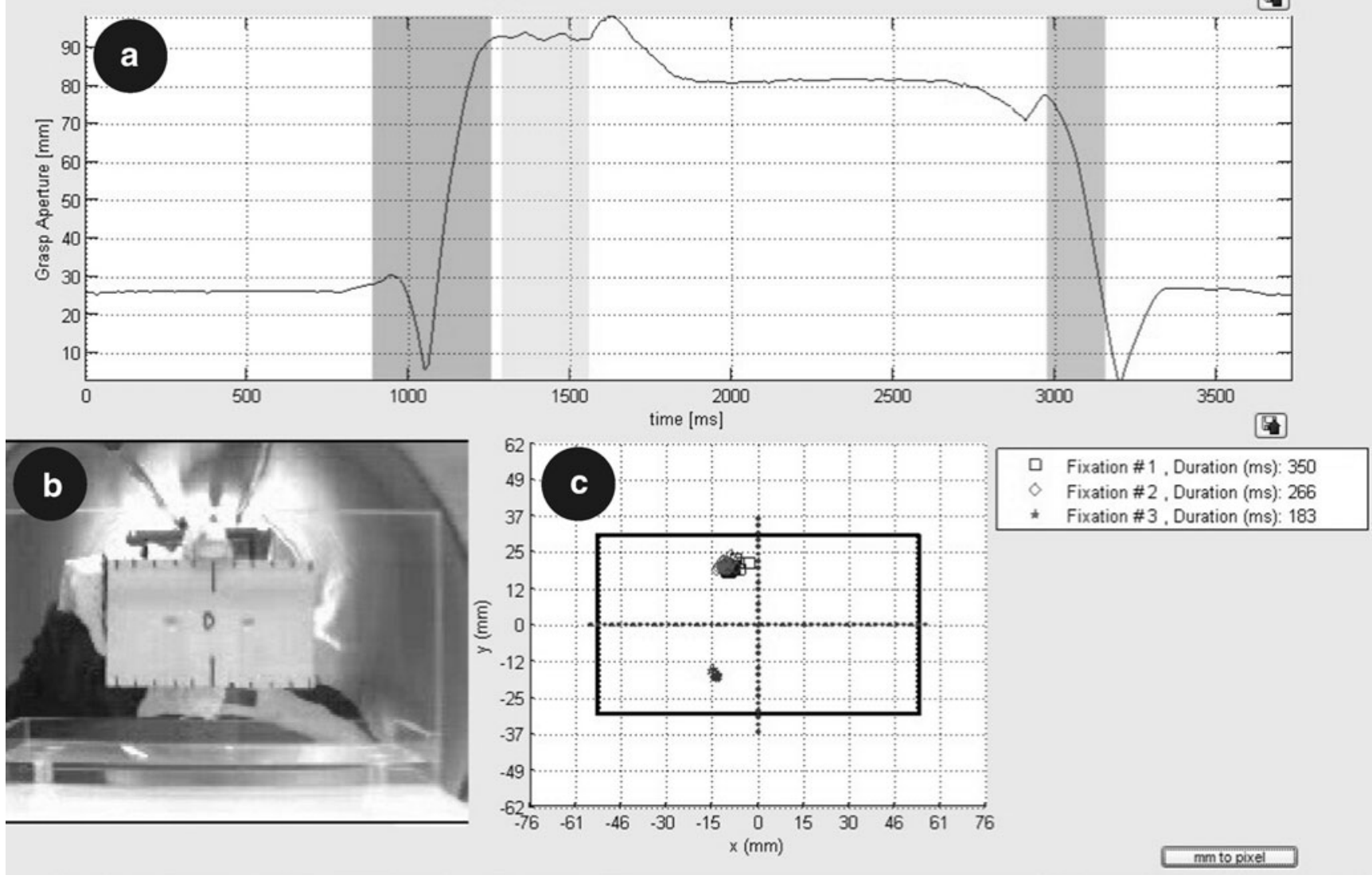

Fig. 3 A screen shot of the BIRA analysis graphical user interface (GUI) during data analysis of one typical trial. The letters A, B, and C were added to label BIRA's different data displays and are not visible on the actual GUI. The graph labelled a shows the timing of grasp aperture (line plot) and eye fixations (shaded areas overlaid onto grasp data). The video screen shot labelled $\mathbf{b}$ shows video data of the grasp

\section{Results}

\section{Grasp kinematics}

A representative aperture profile is demonstrated in Fig. 4. In a typical grasp, participants open their hand smoothly to a peak (or maximum) aperture and close it as the hand approaches the object. In this example, a decrease in aperture was observed prior to the increase during reaching. The interactive design of BIRA allows data points of any graph of kinematic data to be selected and the corresponding video data to be reviewed. Using this tool, we were able to observe a tilt in index finger position, which resulted in the tips of the index finger and thumb moving closer together.

The observed hand behaviour was consistent with what has been seen in previous grasping studies (Gentilucci et al., 1991; during the same trial recorded from the video camera positioned behind the block. The graph labelled c displays the positional information of the gaze data, as represented by the scatterplots superimposed onto a 2-D schematic of the block. Note that the dimensions of the schematic block can be specified to correspond to the dimensions of the actual block used in the experiment

Jakobson \& Goodale, 1991; Jeannerod, 1986; Paulignan, Jeannerod, MacKenzie, \& Marteniuk, 1991). Participants demonstrated accurate grip scaling, opening their hand the widest for the largest block (Fig. 5). This observation was confirmed statistically using a repeated measures ANOVA, which yielded a significant difference for maximum grip aperture as a function of block size $[F(2,6)=8.88, p<.05]$. Tukey's HSD comparison tests were conducted for each pair-wise comparison. Significant differences were observed between Blocks 1 and $2(p<.05)$ and 1 and $3(p<.01)$. However, no significant difference was found between Blocks 2 and $3(p>.05)$.

\section{Reach kinematics}

A typical velocity profile is shown in Fig. 6. In a typical reach, participants accelerate smoothly to a peak (or 
maximum) velocity and then decelerate as their hand approaches the object to be grasped. Once the grasp is over, a new velocity peak will be seen as the hand travels back to its "home" position. From the velocity profiles, BIRA allows traditional reach kinematics to be observed. As examples, the average onset time to start of the hand movement of all participants in all blocks was found to be $0.7 \pm 0.05 \mathrm{~s}$, and the average peak velocity was $0.48 \pm 0.02 \mathrm{~m} / \mathrm{s}$.

\section{Gaze fixations}

The detected first fixation locations are plotted in Fig. 7. The locations of the first fixations fell along the vertical midline of the object, with no significant difference in laterality across blocks $[F(2,4)=0.043, p>.05]$. The vertical position shifted upwards as block size increased $[F(2,4)=18.599, p<.01]$. This behaviour is consistent with measurements observed outside the MRI environment in previous work in our laboratory (Desanghere \& Marotta, 2008).

Eye-hand interactions

In order to demonstrate the advantages of BIRA's capabilities to integrate independent eye and hand data during analysis, we overlaid plots of the fixation and grasp locations from a single participant during grasping of Block 2 (Fig. 8). In general, the participant's first fixation was located near the midline of the object and the grasp site of index finger. The mean fixation location was $2.8 \mathrm{~mm}$ from the horizontal centre of the block and $28.2 \mathrm{~mm}$ from the vertical midline (1.8 $\mathrm{mm}$ from the top edge of the block). The mean horizontal values of index finger placement on Block 2 were found to be $11.2 \mathrm{~mm}$ left of the middle by the ShapeClaw and $2.8 \mathrm{~mm}$ left of the midline of the object by

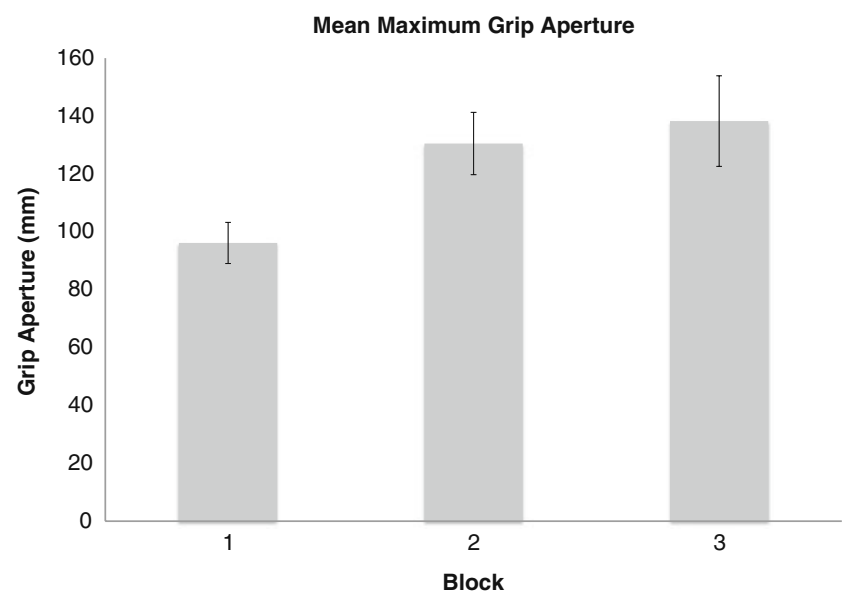

Fig. 5 Mean maximum grip apertures obtained during grasping of Blocks 1, 2, and 3. These results show scaling of maximum grip apertures as a function of block height. Error bars indicate standard errors

the video. Grasp stability was determined by the distance from the mean grasp line to the centre of mass $(\mathrm{COM})$ of Block 2. The distance of the mean grasp line from the COM of Block 2 obtained by the ShapeClaw (indicated by the solid line in Fig. 8) was $3.0 \mathrm{~mm}$ left of the COM, whereas the mean grasp line obtained through measurements made using the video (indicated by the dashed line in Fig. 8) was $0.9 \mathrm{~mm}$ to the right of the COM.

We also used BIRA to analyse the time course of synchronized eye position and grip aperture data obtained from one typical trial (Fig. 9). These synchronized data show the participant open her eyes and make a saccade within the first second of the trial, followed by eye fixation during the reach and grasp. Grip aperture data show that maximum grip aperture was $91 \mathrm{~mm}$ (B in Fig. 9) at approximately $1,800 \mathrm{~ms}$ from the start of the trial, and

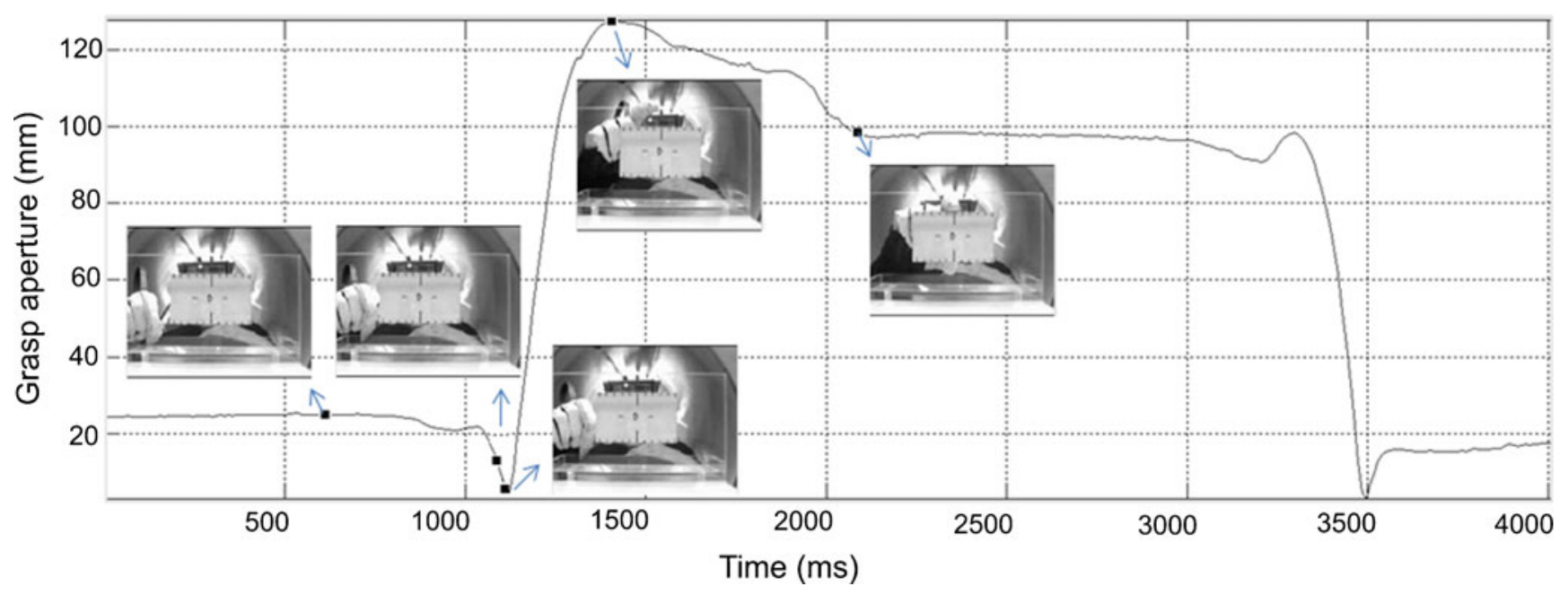

Fig. 4 A representative plot of grip aperture over the course of one trial. In BIRA, a data point can be selected and the corresponding video reviewed frame-by-frame. In the figure, screen shots of the video are overlaid onto the plot with the time point identified 


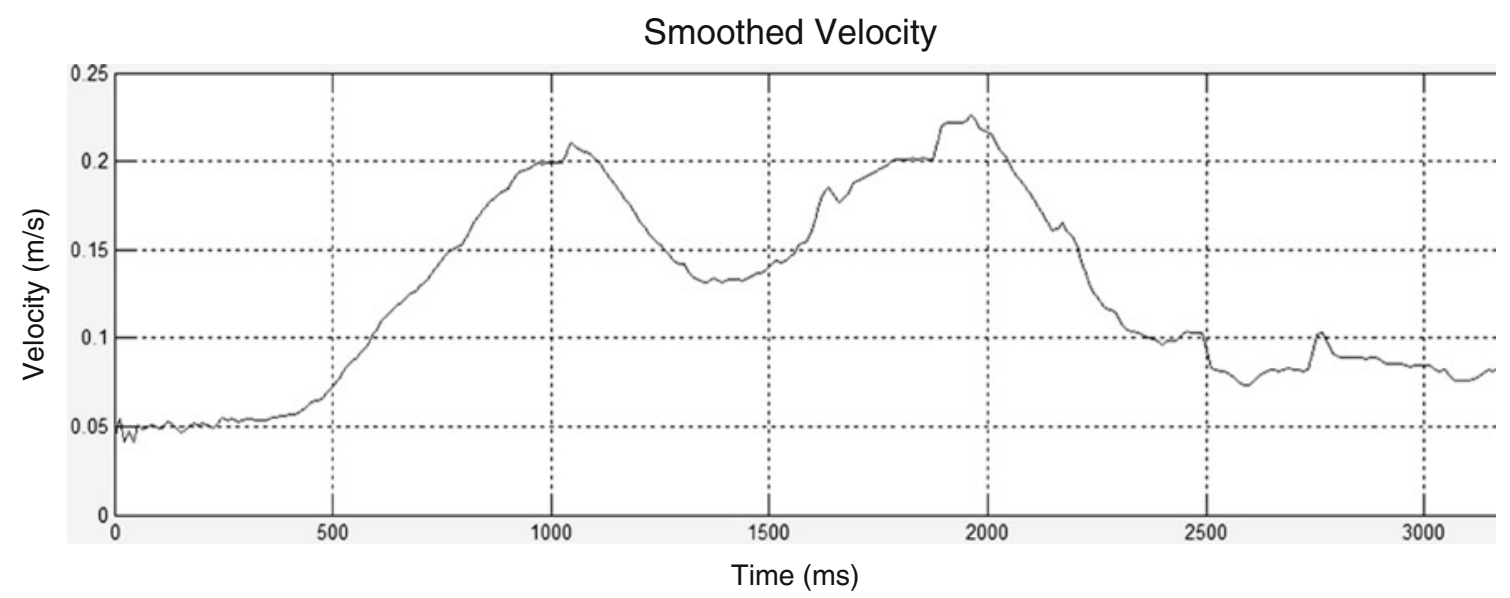

Fig. 6 A typical velocity profile of 1 participant during one grasping trial. The first peak in velocity corresponds to the movement of the hand towards the block. The second peak corresponds to the movement of the hand back to the "home" position

aperture during grasping of the block was $49 \mathrm{~mm}$ from 2,400 to 3,400 ms (between $\mathrm{C}$ and D in Fig. 9).

\section{Discussion}

The present article describes a specialized software package we developed, the BIRA program, which was designed to record, integrate, and analyse eye and hand kinematic data acquired simultaneously from two commercially available tracking systems, in addition to video-based recording. To demonstrate this, we presented the available kinematic data in a simple grasping task conducted with healthy participants. The purpose of this proof-of-concept experiment was to show the types of behavioural information that would be obtainable in an MRI setting. Since the equipment utilized in the present study is commercially available, each of these types of behavioural data can already be acquired individually. However, a literature review of reaching and grasping fMRI studies reveals that they are seldom implemented. Importantly, we present the ability to collect these comprehensive kinematic grasping data using an MRI-friendly method. This software development provides a novel and exciting way to integrate these data, allowing for a comprehensive picture of eye and hand data in an MRI-compatible design.
Fig. 7 Plot of all of the observed first fixation locations during grasping of three blocks. Block dimensions are identified by the solid outlines. Dashed lines represent vertical and horizontal midlines. Diamonds represent first fixations during the presentation of Block 1 . Squares represent first fixations during the presentation of Block 2. Triangles represent the first fixations during the presentation of Block 3

\section{First fixation locations}

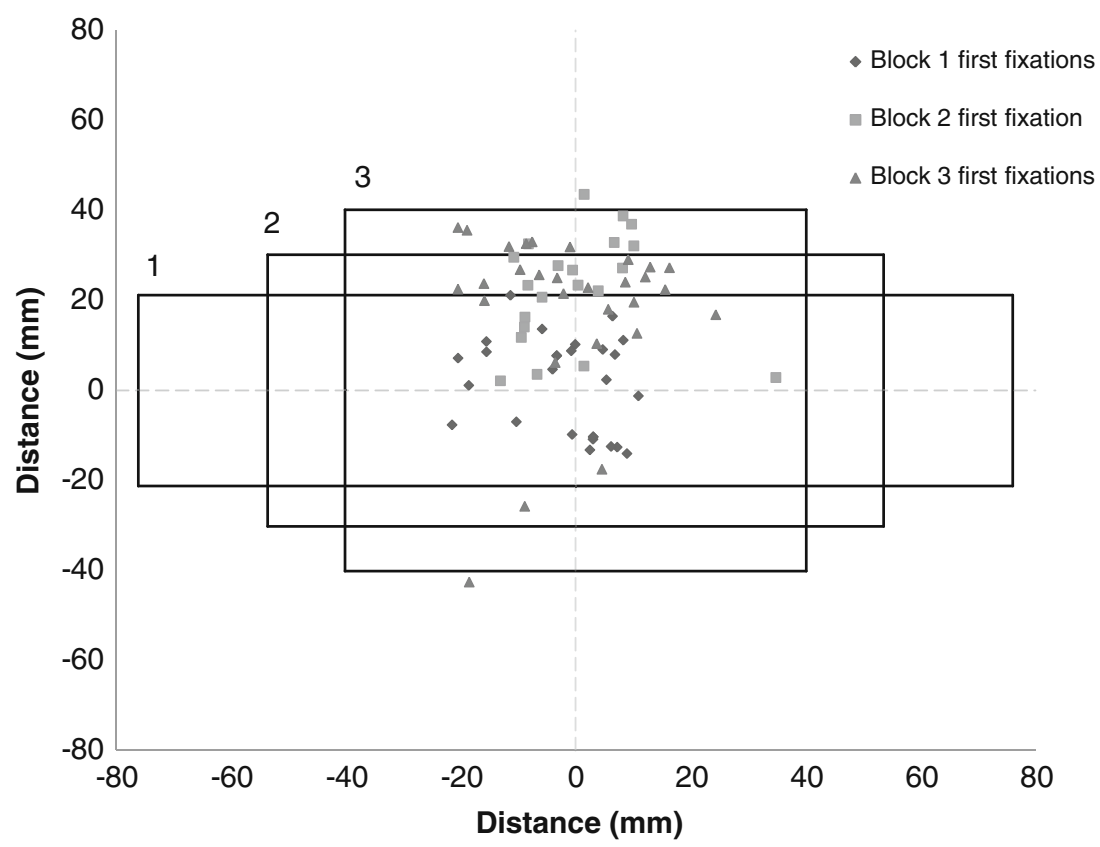


First fixations and grasp data during block 2

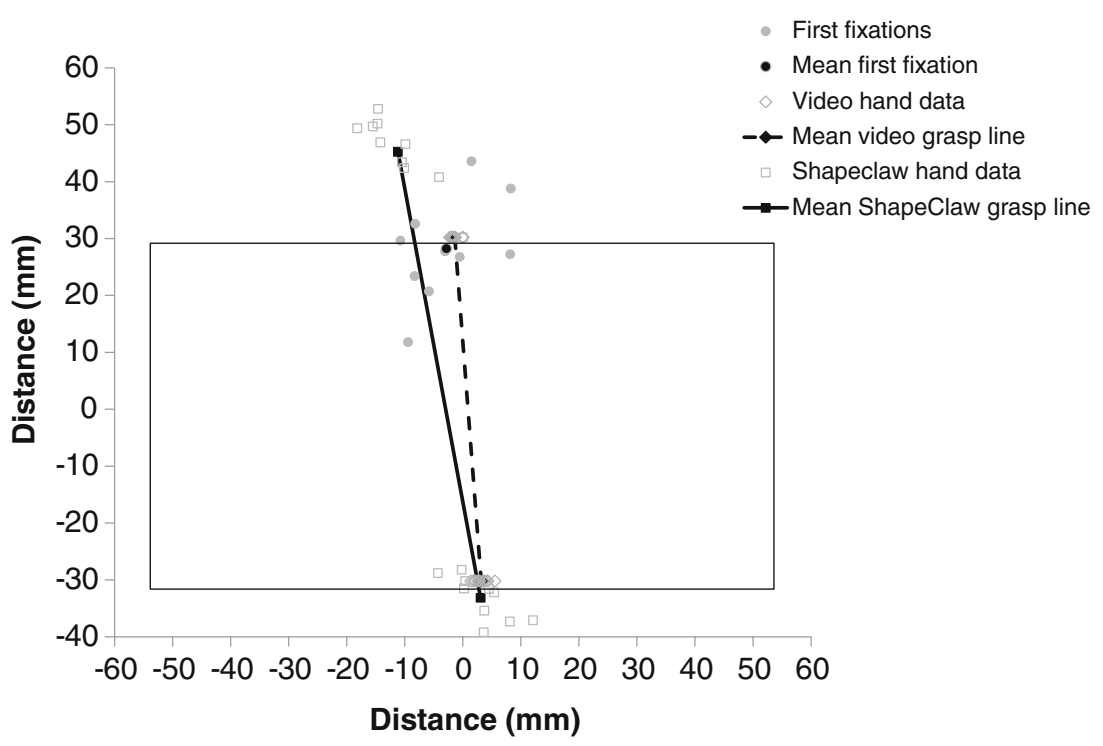

Fig. 8 Eye and hand kinematic data acquired from 10 grasps of Block 2 from a representative participant. Block 2 is represented by the rectangular 2-D schematic outline. First fixations for each grasp are shown by the individual grey circles. Mean fixation position is indicated by the black circle. Index finger and thumb locations for each grasp as determined by video data are represented by the diamonds. Index finger

Our analysis with BIRA revealed hand behaviour consistent with previous findings from grasping studies (Gentilucci et al., 1991; Jakobson \& Goodale, 1991; Jeannerod, 1986; Paulignan et al., 1991). Maximum grip aperture increased with increasing block size. Significant and thumb locations for each grasp as determined by ShapeClaw data are represented by the grey squares. The mean grasp lines, derived by drawing a line connecting the mean index finger position with the mean thumb position on the block, are shown. The dashed line indicates the mean grasp line of the video data, and the solid line indicates the mean grasp line of the ShapeClaw data
Fig. 9 Plot of eye position and grip aperture with respect to time from one typical trial. A median filter was applied to the data. The blue-coloured scale on the left ordinate axis measures grip aperture (blue line plot) in millimetres. Events of hand behaviour during the trial are labelled as (a) the start of hand movement, (b) maximum grip aperture, (c) grasp of the block, (d) release of the block, and (e) return of hand to the "home" position. The green- and redcoloured scales on the right ordinate axis measure horizontal eye position (green line plot) and vertical eye position (red line plot) in a coordinate system with 0,0 representing the centre of the block. Shaded regions indicate fixations of the eye position

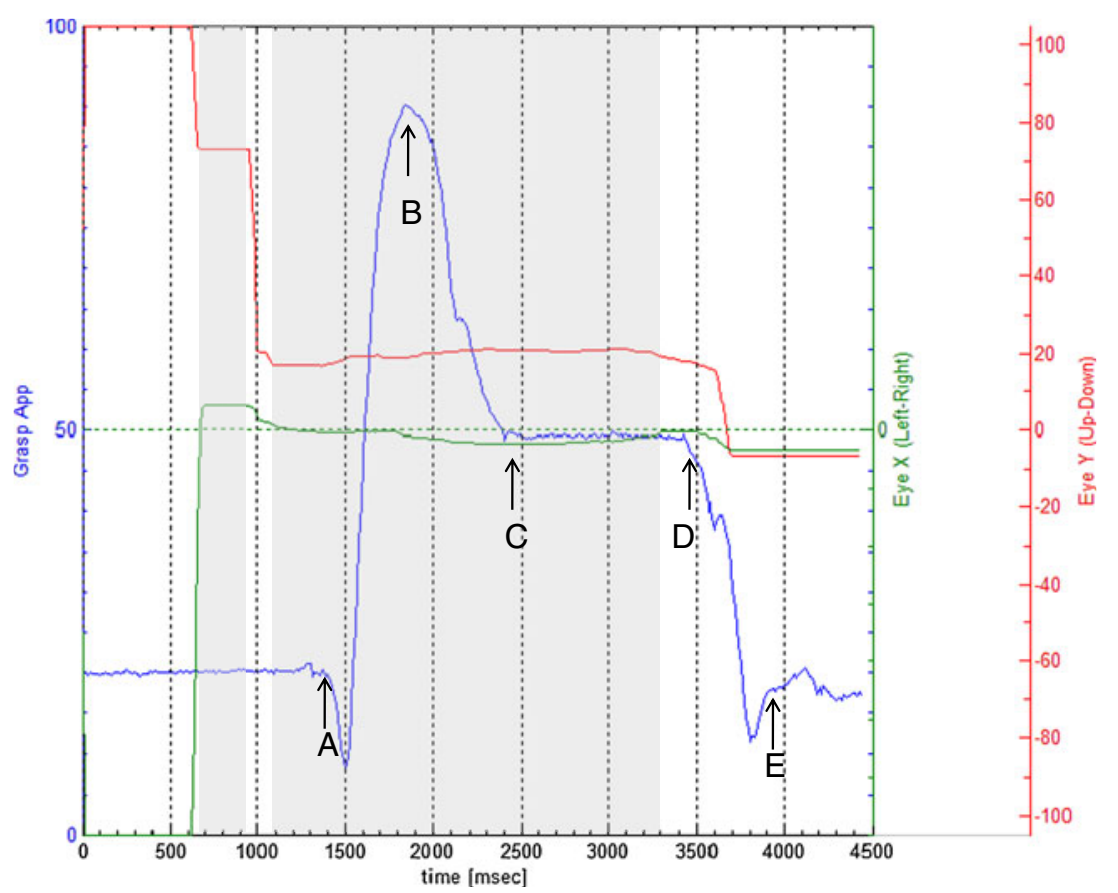

differences in maximum grip aperture were observed between Blocks 1 and 2 and Blocks 1 and 3, but not between Blocks 2 and 3. Larger variations were observed during Blocks 2 and 3, which may be due, in part, to the nature and sensitivity of the ShapeClaw equipment. We 
found ShapeClaw measurements of aperture during grasping of the block (a final aperture that should be equal to block size) to be generally within $20 \mathrm{~mm}$ (data not shown). This finding can be explained by the structure of the ShapeClaw, with tapelets sitting on the superior surface of the index finger and thumb and held in place by a golf glove. This is why, in Fig. 8, index finger positions appear 10-20 mm above the block, since the coordinate is taken from above the index finger. It follows, therefore, that this measurement is influenced by hand shaping. For example, if a block is grasped by the edge so that the index finger tilts up, the coordinate will have a greater $y$ value than if the participant clamps down on the block. However, in the example provided in Fig. 8, the lateral positions of the index finger according to the ShapeClaw measurements and their verification on video are within $8 \mathrm{~mm}$ of each other. From these trials, we determined that the data from the ShapeClaw are very sensitive to finger position. One of the advantages of BIRA is the ability to modify the equipment utilized. With the development of new technologies, the program can be adapted without having to replace all components of the experimental setup (i.e., the eye-tracking and video equipment).

Inspection of the eye kinematic data revealed behaviour consistent with our recent behavioural study investigating gaze strategies while grasping (Desanghere \& Marotta, 2008). We have previously demonstrated that first fixations land near the superior midline of an object prior to hand placement, with subsequent fixations drifting downwards. When we examine eye behaviour across the whole trial in conjunction with hand behaviour, we can observe the gaze direction drifting downwards after the grasp (Fig. 8). This analysis is a demonstration of BIRA's ability to integrate eye and hand data. Without BIRA software, these two sets of kinematic data would be evaluated separately and would be subject to timing errors. BIRA software easily plots both hand and eye kinematic information together to allow for a comprehensive view of behaviour.

Even though the eye- and hand-tracking systems we used in the present study could be utilized individually and the data they obtain analysed independently, BIRA offers a readily available system for integrating and analysing data from any tandem of eye- and hand-tracking systems. The use of this multimodal approach allows for the construction of more comprehensive behavioural information, as illustrated in Figs. 8 and 9. Even though this study design had been widely available in a lab setting, to the best of our knowledge such a detailed approach has not been utilized in an MRI environment. The present report is the first step in addressing this gap, by describing the development of software that makes the execution of these studies easier, using available independent systems.
The development of software that uses independent motion-tracking systems offers further advantages. First, it allows laboratories to utilize their existing motiontracking equipment. Second, it allows for replacement of any of the individual motion-tracking systems as more advanced equipment becomes available. For example, our eye-tracking system only offers monocular tracking and requires attaching an additional mirror to the head coil. However, BIRA can easily be adapted to record data from a binocular eye-tracking system.

In addition to being easily adaptable to other tracking systems, BIRA offers several other advantages for visuomotor research. BIRA is a freely available (www. perceptionandaction.com), easy-to-learn system. It is user friendly, with a "point-and-click" graphical interface, and does not require any background in computer programming. However, BIRA's code is open source, allowing researchers to customize BIRA to meet their specific research needs (e.g., adding more kinematic parameters and analyses), and these modifications can be shared amongst the research community. Further, BIRA can run on a common PC laptop, so its portability is only limited by the laboratory's tracking systems. The versatility of BIRA lends itself to a much broader range of applications. For example, BIRA can be used with portable eye- and hand-tracking monitors in non-MRI, applied visuomotor studies outside the laboratory environment (e.g., eye-hand coordination in a driving task). Furthermore, the experimental setup can be modified for the integration of alternative or additional equipment to expand the applications that BIRA could be used for. In the present setup, a modified golf glove was used to secure the ShapeClaw tapelets to the superior surface of the index finger and thumb. With a simple modification to the glove so that the inferior surfaces of the finger tips are exposed, investigations of the influence of tactile input on motor control could also be advanced using the BIRA paradigm.

Notwithstanding other potential experimental applications, BIRA is perfectly suited for fMRI research on visuomotor behaviour. As MRI-compatible eye- and hand-tracking systems become more common, fMRI research will benefit from this kind of integrative method of recording and analysing gaze and kinematic data from separate tracking systems. BIRA's integrative method offers additional sophisticated gaze and hand analyses over existing, equipment-specific software, which can complement fMRI analysis. For example, BIRA's enhanced analyses allow one to eliminate potential confounds of gaze and hand data that might elicit significant differences in brain activation between experimental conditions. Moreover, the gaze and kinematic data obtained by BIRA can be entered as covariates in a general linear model. 


\section{Conclusion}

In the present article, we demonstrated a novel software program that records, integrates, and analyses reliable eyehand kinematic data. Future studies will assess the benefits of using BIRA for the analysis of fMRI studies of reaching and grasping. The application of such a multimodal approach will provide a more comprehensive method for studying visuomotor control and the function of the underlying neural networks.

Author Note This work was supported by a Natural Sciences and Engineering Research Council of Canada (NSERC) Research Tools and Instruments Grant and a Canada Foundation for Innovation (CFI) New Opportunities Grant to J.J.M. J.M.L. is supported by a MHRC research fellowship. B.P.M., L.D., and L.A.B. are recipients of MHRC graduate studentships. We gratefully acknowledge Kris Malisza and the National Research Council, Institute for Biodiagnostics, for the use of their mock MRI scanner.

\section{Appendix}

\section{Calibrating ShapeClaw}

ShapeClaw (Measureand, Fredricton, NB) is a fibre-opticbased hand motion-tracking system. ShapeClaw consists of fibre-optic cables set in two flexible ribbons. The ShapeClaw ribbons are secured to the participant's index finger and thumb by a special glove and run up the entire length of the arm (shown in Fig. 1A). ShapeClaw captures the position and movement of the index finger and thumb, including the orientation and position of the hand and forearm. ShapeClaw's calibration was performed using its native software, the ShapeRecorder program. We describe the calibration procedure below.

ShapeClaw was first calibrated in the "flat tape" position with the ShapeClaw ribbons laid completely flat on the floor. The ShapeClaw was then affixed to the participant using the special glove and arm straps. In addition to the arm straps provided with the ShapeClaw, we also used a nylon armband that covered the whole length of the arm, to further ensure that the ShapeClaw ribbons were secured to the participant. Prior to calibration, measurements of the participant's hand width, wrist width, wrist thickness, arm length, finger and thumb lengths, shoulder breadth, and body girth were entered into ShapeRecorder. ShapeRecorder co-registered these anatomical measurements with the length and position of the ShapeClaw ribbons when they were attached to the participant's hand and arm. ShapeClaw was then calibrated at different hand and finger positions. The sequence of hand/finger positions was hand palm with fingers straight, closed fist, fingers splayed, fingers flat with thumb pointing downward, wrist bent up, wrist bent down, and thumb touching index finger. All positions were done with the arm straight at the participant's side. In an effort to ensure reliable grasp data during data collection, we repeated the calibration sequence before each block of trials.

\section{References}

Abramoff, M. D., Magelhaes, P. J., \& Ram, S. J. (2004). Image processing with ImageJ. Biophotonics International, 11, 36-42.

Brainard, D. H. (1997). The psychophysics toolbox. Spatial Vision, 10, 433-436.

Carey, D. P., Della Sala, S., \& Ietswaart, M. (2002). Neuropsychological perspectives on eye-hand coordination in visually-guided reaching. Progress in Brain Research, 140, 311-327.

Culham, J. C., Cavina-Pratesi, C., \& Singhal, A. (2006). The role of parietal cortex in visuomotor control: What have we learned from neuroimaging? Neuropsychologia, 44, 2668-2684.

Culham, J. C., Danckert, S. L., DeSouza, J. F., Gati, J. S., Menon, R. S., \& Goodale, M. A. (2003). Visually guided grasping produces fMRI activation in dorsal but not ventral stream brain areas. Experimental Brain Research, 153, 180-189.

Desanghere, L., \& Marotta, J. J. (2008). Gaze strategies while grasping: What are you looking at?! [Abstract]. Journal of Vision, 8(6), 495a.

Diedrichsen, J., Hashambhoy, Y., Rane, T., \& Shadmehr, R. (2005). Neural correlates of reach errors. The Journal of Neuroscience, 25, 9919-9931.

Gentilucci, M., Castiello, U., Corradini, M. L., Scarpa, M., Umiltà, C., \& Rizzolatti, G. (1991). Influence of different types of grasping on the transport component of prehension movements. Neuropsychologia, 29, 361-378.

Glover, S. (2004). Separate visual representations in the planning and control of action. The Behavioral and Brain Sciences, 27, 3-24.

Grefkes, C., Ritzl, A., Zilles, K., \& Fink, G. R. (2004). Human medial intraparietal cortex subserves visuomotor coordinate transformation. Neuroimage, 23, 1494-1506.

Jakobson, L. S., \& Goodale, M. A. (1991). Factors affecting higherorder movement planning: A kinematic analysis of human prehension. Experimental Brain Research, 86, 199-208.

Jeannerod, M. (1986). The formation of finger grip during prehension: A cortically mediated visuomotor pattern. Behavioural Brain Research, 19, 99-116.

Levy, I., Schluppeck, D., Heeger, D. J., \& Glimcher, P. W. (2007). Specificity of human cortical areas for reaches and saccades. The Journal of Neuroscience, 27, 4687-4696.

Marotta, J. J., Mraz, R., Black, S. E., \& Graham, S. J. (2007). An fMRI investigation of grasping in the elderly and in stroke. Neuroimage, 36, S51.

Ogawa, K., Inui, T., \& Sugio, T. (2006). Separating brain regions involved in internally guided and visual feedback control of moving effectors: An event-related fMRI study. Neuroimage, 32, 1760-1770.

Paulignan, Y., Jeannerod, M., MacKenzie, C., \& Marteniuk, R. (1991). Selective perturbation of visual input during prehension movements: 2 . The effects of changing object size. Experimental Brain Researc, 87, 407-420.

Pelli, D. G. (1997). The VideoToolbox software for visual psychophysics: Transforming numbers into movies. Spatial Vision, 10, 437-442.

Rocca, M. A., Gatti, R., Agosta, F., Tortorella, P., Riboldi, E., Broglia, P., et al. (2007). Influence of body segment position during in-phase and antiphase hand and foot movements: A kinematic and functional MRI study. Human Brain Mapping, 28, 218-227.

Salvucci, D. D., \& Goldberg, J. H. (2000). Identifying fixations and saccades in eye-tracking protocols. In Proceedings of the 2000 symposium on eye tracking research and applications symposium (pp. 71-78). New York: ACM Press.

Schaechter, J. D., Stokes, C., Connell, B. D., Perdue, K., \& Bonmassar, G. (2006). Finger motion sensors for fMRI motor studies. Neuroimage, 31, 1549-1559. 